\title{
Solvability of differential algebraic equations and inequalities: an algorithm
}

\author{
S. T. Glad* \\ Department of Electrical Engineering \\ Linköping University \\ S-581 83 Linköping, Sweden \\ Tel (+46) 13 281308, Fax (+46) 13282622 \\ e-mail: torkel@isy.liu.se
}

\begin{abstract}
Summary
The existence of real solutions to polynomial systems of implicit differential equations, differential inequations and differential inequalities is considered. A transformation to a standard form is achieved using methods of differential algebra. The problem then reduces to a problem in real (non-differential) algebra.
\end{abstract}

\section{Introduction}

When modeling physical systems one often arrives at a mixture of equations in a number of physical variables and their derivatives, see e. g. [7] and [8]. The model might only be valid for certain values of the physical variables or one might want to study it only in a certain range. This could be expressed by inequalities or inequations. An obvious question is then whether there exists a solution that satisfies all equations, inequations and inequalities. Also one would like to transform the system to some standard form which facilitates the computation of such a solution.

For the case of a system of polynomial equations and inequations

$$
f_{1}=0, \ldots, f_{n}=0 ; \quad g_{1} \neq 0, \ldots, g_{m} \neq 0
$$

in a number of variables and their derivatives, there is an algorithm by Seidenberg, [13] to decide solvability using successive elimination of variables. The algorithm does not allow the restriction to real-valued solutions that one would want in most physical models, however.

In the work by Ritt, [12], a standard form, the so called characteristic set is introduced, which permits investigation of solvability but gives no direct information about real solutions. Also the Ritt algorithm requires factorizations of high complexity. A further investigation of algorithmic aspects is given in [5].

For polynomial systems of equations, inequations and inequalities without any derivatives, the theory of real algebra, [10], [3], gives algorithmic methods for

*This work was supported by the Swedish Research Council for Engineering Sciences (TFR), which is gratefully acknowledged. deciding the existence of real solutions. A possible algorithm is cylindrical algebraic decomposition., [1], [2].

In this paper we will look at systems of equations, inequations and strict inequalities:

$$
\begin{gathered}
f_{1}=0, \ldots, f_{n}=0, \quad g_{1} \neq 0, \ldots, g_{m} \neq 0 \\
h_{1}<0, \ldots, h_{q}<0
\end{gathered}
$$

The $f_{i}, g_{i}$ and $h_{i}$ are differential polynomials in some variables $y_{1}, \ldots, y_{N}$, i. e. they are polynomials in those variables and a finite number of their derivatives. The coefficients will typically be rational numbers, but may in principle come from any real field.

\section{Basic differential algebraic concepts}

We will use concepts from differential algebra to construct an algorithm. An introduction to differential algebra is given in [6]. The basic references are [12], [11]. Algorithmic aspects are discussed in [9] and in $[4],[5]$.

As discussed above we will be interested in systems described by differential polynomials, i.e. polynomials in certain variables and their derivatives. The derivatives will be denoted by dots or the derivative order in parenthesis:

$$
u, \quad \dot{u}, \quad \ddot{u}, \quad u^{(3)}, \ldots
$$

A fundamental concept for the algorithmic aspects of differential algebra is ranking. This is a total ordering of all variables and their derivatives. Examples involving two variables are

$$
u<y<\dot{u}<\dot{y}<\ddot{u}<\ddot{y}<\cdots
$$


In the latter case one could perform a pseudo-

$$
u<\dot{u}<\ddot{u}<\cdots<y<\dot{y}<\ddot{y}<\cdots
$$

where $<$ denotes "is ranked lower than". Any ranking is possible provided it satisfies two conditions:

$$
\begin{gathered}
u^{(\mu)}<u^{(\mu+\sigma)} \\
u^{(\mu)}<y^{(\nu)} \Rightarrow u^{(\mu+\sigma)}<y^{(\nu+\sigma)}
\end{gathered}
$$

for all variables $u$ and $y$, all nonnegative integers $\mu$ and $\nu$, and all positive integers $\sigma$. The highest ranking variable or derivative of a variable in a differential polynomial is called the leader.

The ranking of variables gives a ranking of differential polynomials. They are simply ranked as their leaders. If they have the same leader, they are considered as polynomials in their leader and the one of lowest degree is ranked lower.

Let $A, B$ be two differential polynomials and let $A$ have the leader $v$. Then $B$ is said to be reduced with respect to $A$ if there is no derivative of $v$ in $B$ and if $B$ has lower degree than $A$ when both are regarded as polynomials in $v$.

A set

$$
A_{1}, \ldots, A_{p}
$$

of differential polynomials is called auto-reduced if all the $A_{i}$ are pairwise reduced with respect to each other. Normally auto-reduced sets are ordered so that $A_{1}, . ., A_{p}$ are in increasing rank.

Auto-reduced sets are ranked as follows. Let $\mathbf{A}=$ $A_{1}, \ldots, A_{r}$ and $\mathbf{B}=B_{1}, \ldots, B_{s}$ be two ordered autoreduced sets. $\mathbf{A}$ is ranked lower if either there is an integer $k, 0 \leq k \leq \min (s, r)$ such that

$$
\begin{aligned}
& \operatorname{rank} A_{j}=\operatorname{rank} B_{j}, \quad j=0, \ldots, k-1 \\
& \operatorname{rank} A_{k}<\operatorname{rank} B_{k}
\end{aligned}
$$

or else if $r>s$ and

$$
\operatorname{rank} A_{j}=\operatorname{rank} B_{j}, \quad j=0, \ldots, s
$$

A characteristic set for a given set of differential polynomials is an auto-reduced subset such that no other auto-reduced subset is ranked lower.

The separant $S_{A}$ of a differential polynomial $A$ is the partial derivative of $A$ with respect to the leader, while the initial $I_{A}$ is the coefficient of the highest power of the leader in $A$.

If a differential polynomial $f$ is not reduced with respect to another differential polynomial $g$, then either $f$ contains some derivative of the leader $u_{g}$ of $g$ or else $f$ contains $u_{g}$ to a higher power. In the former case one could differentiate $g$ a suitable number (say $\sigma$ ) of times and perform a pseudo-division to remove that derivative, giving a relation

$$
S^{\nu} f=Q g^{(\sigma)}+R
$$

where $S$ is the separant of $g$ and $R$ does not contain the highest derivative of $u_{g}$ which is present in $f$. division of $f$ by $g$ getting

$$
I^{\nu} f=Q g+R
$$

where $I$ is the initial of $g$ and $R$ is reduced with respect to $g$.

The following property is important for the finiteness of the algorithms that we are going to present.

Proposition 1 A sequence of derivatives, each one ranked lower than the preceding one, can only have finite length.

Proof. Let $y_{1}, \ldots, y_{p}$ denote all the variables whose derivatives appear anywhere in the sequence. For each $y_{j}$ let $\sigma_{j}$ denote the order of the first appearing derivative. There can then be only $\sigma_{j}$ lower derivatives of $y_{j}$ in the sequence. The total number of elements is thus bounded by $\sigma_{1}+\cdots+\sigma_{p}+p$.

A direct consequence is

Proposition 2 A sequence of characteristic sets, each one ranked lower than the preceding one, can only have finite length.

\section{Equations and inequations}

Consider to begin with a system of the form

$$
f_{1}=0, \ldots, f_{n}=0, \quad g_{1} \neq 0, \ldots, g_{m} \neq 0
$$

where the $f_{i}$ and $g_{i}$ are polynomials in the variables

$$
y_{1}, \ldots, y_{N}
$$

and their derivatives. The basic algorithm is the following.

\section{Algorithm FG}

Input: An ordered pair $(F, G)$ of sets

$$
F=\left\{f_{1}, \ldots, f_{n}\right\}, \quad G=\left\{g_{1}, \ldots, g_{m}\right\}
$$

where the $f_{i}$ and $g_{i}$ are differential polynomials corresponding to equations and inequations respectively.

1. Compute a characteristic set

$$
\mathbf{A}=\left\{A_{1}, \ldots, A_{p}\right\}
$$

of $F$.

2. If $F \backslash \mathbf{A}$ is non-empty, then go to 5 .

3. If $S_{A} \in G, I_{A} \in G$ for all $A \in \mathbf{A}$ then Finished. Output: $(F, G)$.

4. For some $A \in \mathbf{A}$, either $p:=S_{A}$ or $p:=I_{A}$ so that $p \notin G$.

Split. Output:

$$
(F \cup\{p\}, G) \quad(F, G \cup\{p\})
$$


5. Let $f_{k}$ be the highest unreduced (with respect to A) element of $F$. Then from (2), (3), there is an equation

$$
p^{\nu} f_{k}=Q f_{j}^{(\sigma)}+R
$$

where $f_{j}$ is an element of $\mathbf{A}$, and either $p=S_{f_{j}}$ or $p=I_{f_{j}}$. If $p \notin G$ then go to 8 .

6. If $R=0$ then

$$
F:=F \backslash f_{k}
$$

and go to 1 .

7.

$$
F:=\left(F \backslash\left\{f_{k}\right\}\right) \cup\{R\}
$$

and go to 1 .

8. Split. Output:

$$
(F \cup\{p\}, G), \quad(F, G \cup\{p\})
$$

Proposition 3 The algorithm $F G$ will reach one of the points marked "Finished" or "Split" after a finite number of steps.

Proof. The only possible loop is via step 6 or step 7 to step 1. This involves either the removal of a polynomial or its replacement with one that is reduced with respect to $\mathbf{A}$ or has its highest unreduced derivative removed. If $R$ is reduced, then it is possible to construct a lower auto-reduced set. An infinite loop would thus contradict either Proposition 1 or Proposition 2.

Proposition 4 If the algorithm FG receives the pair $(F, G)$ of equations and inequations and returns the two pairs $\left(F_{1}, G_{1}\right),\left(F_{2}, G_{2}\right)$, then

$$
(F, G) \Leftrightarrow\left(F_{1}, G_{1}\right) \operatorname{or}\left(F_{2} \cdot G_{2}\right)
$$

in the sense that an element of a given differential algebraic field satisfies the equations $F$ and the inequations $G$ if and only if it satisfies either $F_{1}, G_{1}$ or $F_{2}, G_{2}$.

Proof. The set $F$ is changed at either step 6 or step 7. If these steps are reached, then we have

$$
p^{\nu} f_{k}=Q f_{j}^{(\sigma)}+R
$$

with $p$ belonging to the set of inequations $G$ that have to be satisfied. The problems

$$
\begin{aligned}
& f_{1}=0, \ldots, f_{k}=0, \ldots, f_{n}=0 ; \quad \prod_{g \in G} g \neq 0 \\
& f_{1}=0, \ldots, R=0, \ldots, f_{n}=0 ; \quad \prod_{g \in G} g \neq 0
\end{aligned}
$$

are then equivalent. At the splittings at step 4 or step 8 the equivalence is obvious.
If the algorithm $\mathrm{FG}$ splits the pair $(F, G)$ into two pairs $\left(F_{1}, G_{1}\right)$ and $\left(F_{2}, G_{2}\right)$, then the algorithm can again be used on each pair. If there is a new split, the algorithm can again be used on each pair. In this way a tree structure is generated where each node corresponds to a split generated by the algorithm. If the algorithm reaches "Finished" at step 3, then that branch of the tree is terminated.

Proposition 5 The tree generated by the algorithm FG in the manner described above is finite and each branch terminates with a pair $(F, G)$ such that $F$ is an auto-reduced set and each separant and initial of $F$ belongs to $G$.

Proof. Consider a pair $\left(F_{1}, G_{1}\right)$ generated at one node of the tree and a pair $\left(F_{2}, G_{2}\right)$ generated at a lower node. Then, either the lowest auto-reduced set of $F_{2}$ is strictly lower then the one of $F_{1}$, or else $F_{1}=$ $F_{2}$. In the latter case $G_{2}$ has been obtained from $G_{1}$ by adding one or more elements. Since only a finite number of elements can be added to $G$ for a fixed $F$, an infinite number of nodes in the tree can only be generated by a violation of Proposition 2 . The remainder of the proposition follows from the fact that a branch of the tree can only be terminated by algorithm FG reaching "Finished" at step 3.

\section{A reduced form}

Now consider a system which also includes strict inequalities:

$$
\begin{gathered}
f_{1}=0, \ldots, f_{n}=0, \quad g_{1} \neq 0, \ldots, g_{m} \neq 0 \\
h_{1}<0, \ldots, h_{q}<0
\end{gathered}
$$

We assume that algorithm FG has been used for the equations and inequations so that $f_{1}, \ldots, f_{n}$ form an auto-reduced set whose separants and initials are among $g_{1}, . ., g_{m}$.

Proposition 6 The system (5) is equivalent to a modified system

$$
\begin{gathered}
f_{1}=0, \ldots, f_{n}=0, \quad \tilde{g}_{1} \neq 0, \ldots, \tilde{g}_{m} \neq 0 \\
\tilde{h}_{1}<0, \ldots, \tilde{h}_{q}<0
\end{gathered}
$$

where all $g_{i}$ and $h_{i}$ are reduced with respect to $f_{1}, \ldots, f_{n}$.

Proof. For each $g_{k}$ that is not reduced we can write

$$
g_{k} \prod_{i} S_{i}^{\nu_{i}} I_{i}^{\mu_{i}}=\tilde{g}_{k}+\sum_{i j} Q_{i j} f_{i}^{(j)}
$$

where $\tilde{g}_{k}$ is the remainder of $g_{k}$ with respect to the auto-reduced set $f_{1}, \ldots, f_{n}$ and the $S_{i}$ and $I_{i}$ are the separants and initials. Since these polynomials are among those which are specified to be nonzero and the $f_{i}$ are specified to be zero, it is clear that $g_{k} \neq 0$ 
is equivalent to $\tilde{g}_{k} \neq 0$. Also $\tilde{g}_{k}$, being a remainder, is reduced with respect to $f_{1}, . ., f_{n}$.

For each $h_{k}$ one has similarly a relation

$$
h_{k} \prod S_{i}^{\nu_{i}} I_{i}^{\mu_{i}}=\hat{h}_{k}+\sum Q_{i j} f_{i}^{(j)}
$$

where the remainder $\hat{h}_{k}$ is reduced. Multiplying by as suitable number of separants and initials this can be written

$$
h_{k} \prod S_{i}^{\tilde{\nu}_{i}} I_{i}^{\tilde{\mu}_{i}}=\tilde{h}_{k}+\sum \tilde{Q}_{i j} f_{i}^{(j)}
$$

where the integers $\tilde{\nu}_{i}$ and $\tilde{\mu}_{i}$ are even and

$$
\tilde{h}_{i}=\hat{h}_{k} \prod S_{i}^{\sigma_{i}} I_{i}^{\tau_{i}}
$$

is still reduced with respect to $f_{1}, \ldots, f_{n}$. Since $h_{k}$ and $\tilde{h}_{k}$ differ only by a strictly positive factor when all inequations and equations are satisfied, it follows that $h_{k}<0$ and $\tilde{h}_{k}<0$ are equivalent.

\section{Solvability}

From the previous sections it follows that to determine the existence of a real solution to (1) one has to solve that problem for a number of systems

$$
\begin{gathered}
f_{1}=0, \ldots, f_{n}=0, \quad g_{1} \neq 0, \ldots, g_{m} \neq 0 \\
h_{1}<0, \ldots, h_{q}<0
\end{gathered}
$$

where $f_{1}, \ldots, f_{n}$ is an auto-reduced set whose separants and initials are among the $g_{i}$, and where all $g_{i}$ and $h_{i}$ are reduced with respect to $f_{1}, . ., f_{n}$.

To analyze the problem, the original physical variables are replaced by variables $z_{1}, . ., z_{n}$ and $u_{1}, . ., u_{p}$ $(p=N-n)$ in such a way that the leader of $f_{i}$ is the derivative $z_{i}^{\left(\nu_{i}\right)}$ for some $\nu_{i}$. We introduce the notation

$$
\begin{aligned}
Z_{i}^{\nu} & =\left\{z_{i}, \dot{z}_{i}, \ddot{z}_{i}, \ldots, z_{i}^{(\nu-1)}, z_{i}^{(\nu)}\right\} \\
U & =\left\{u_{1}, \ldots, u_{1}^{\left(\sigma_{1}\right)}, \ldots, u_{p}^{\left(\sigma_{p}\right)}\right\}
\end{aligned}
$$

where $\sigma_{i}$ is the highest derivative of $u_{i}$. The system (7) can then be written

$$
\begin{aligned}
f_{1}\left(Z_{1}^{\nu_{1}}, Z_{2}^{\nu_{2}-1}, Z_{3}^{\nu_{3}-1}, \ldots, Z_{n}^{\nu_{n}-1}, U\right) & =0 \\
f_{2}\left(Z_{1}^{\nu_{1}}, Z_{2}^{\nu_{2}}, Z_{3}^{\nu_{3}-1}, \ldots, Z_{n}^{\nu_{n}-1}, U\right) & =0 \\
\vdots & \\
f_{n}\left(Z_{1}^{\nu_{1}}, Z_{2}^{\nu_{2}}, Z_{3}^{\nu_{3}}, \ldots, Z_{n}^{\nu_{n}}, U\right) & =0 \\
g_{1}\left(Z_{1}^{\nu_{1}}, Z_{2}^{\nu_{2}}, Z_{3}^{\nu_{3}}, \ldots, Z_{n}^{\nu_{n}}, U\right) & \neq 0 \\
\vdots & \\
g_{m}\left(Z_{1}^{\nu_{1}}, Z_{2}^{\nu_{2}}, Z_{3}^{\nu_{3}}, \ldots, Z_{n}^{\nu_{n}}, U\right) & \neq 0 \\
h_{1}\left(Z_{1}^{\nu_{1}}, Z_{2}^{\nu_{2}}, Z_{3}^{\nu_{3}}, \ldots, Z_{n}^{\nu_{n}}, U\right) & <0 \\
\vdots & \\
h_{q}\left(Z_{1}^{\nu_{1}}, Z_{2}^{\nu_{2}}, Z_{3}^{\nu_{3}}, \ldots, Z_{n}^{\nu_{n}}, U\right) & <0
\end{aligned}
$$

We now introduce the following sets of variables in two indices:

$$
\begin{gathered}
Z_{i, \nu}=\left\{z_{i, 0}, z_{i, 1}, \ldots, z_{i, \nu-1}, z_{i, \nu}\right\} \\
V=\left\{u_{1,0}, \ldots, u_{1, \sigma_{1}}, \ldots, u_{p, \sigma_{p}}\right\}
\end{gathered}
$$

Together with (10) we can then consider the purely algebraic system of equations, inequations and inequalities.

$$
\begin{aligned}
f_{1}\left(Z_{1, \nu_{1}}, Z_{2, \nu_{2}-1}, Z_{3, \nu_{3}-1}, \ldots, Z_{n, \nu_{n}-1}, V\right) & =0 \\
f_{2}\left(Z_{1, \nu_{1}}, Z_{2, \nu_{2}}, Z_{3, \nu_{3}-1}, \ldots, Z_{n, \nu_{n}-1}, V\right) & =0 \\
\vdots & \\
f_{n}\left(Z_{1, \nu_{1}}, Z_{2, \nu_{2}}, Z_{3, \nu_{3}}, \ldots, Z_{n, \nu_{n}}, V\right) & =0 \\
g_{1}\left(Z_{1, \nu_{1}}, Z_{2, \nu_{2}}, Z_{3, \nu_{3}}, \ldots, Z_{n, \nu_{n}}, V\right) & \neq 0 \\
\vdots & \\
g_{m}\left(Z_{1, \nu_{1}}, Z_{2, \nu_{2}}, Z_{3, \nu_{3}}, \ldots, Z_{n, \nu_{n}}, V\right) & \neq 0 \\
h_{1}\left(Z_{1, \nu_{1}}, Z_{2, \nu_{2}}, Z_{3, \nu_{3}}, \ldots, Z_{n, \nu_{n}}, V\right) & <0 \\
& \vdots \\
h_{q}\left(Z_{1, \nu_{1}}, Z_{2, \nu_{2}}, Z_{3, \nu_{3}}, \ldots, Z_{n, \nu_{n}}, V\right) & <0
\end{aligned}
$$

To determine if this system has a real solution can be done using for instance the methods of [1], [2].

\section{Proposition 7 Let}

$$
Z_{1, \nu_{1}}^{o}, \ldots, Z_{p, \nu_{p}}^{o}, V^{o}
$$

solve (13). Then locally around (14) the equations

$$
f_{1}=0, \ldots, f_{n}=0
$$

of (13) are equivalent to a system

$$
\begin{aligned}
z_{1, \nu_{1}} & =\phi_{1}\left(Z_{1, \nu_{1}-1}, \ldots, Z_{n, \nu_{n}-1}, V\right) \\
\vdots & \\
z_{n, \nu_{n}} & =\phi_{n}\left(Z_{1, \nu_{1}-1}, \ldots, Z_{n, \nu_{n}-1}, V\right)
\end{aligned}
$$

Proof. The Jacobian $\partial f_{i} / \partial z_{j, \nu_{j}}$ is a lower triangular matrix from the structure of (13). Its diagonal elements are the separants, which are among the $g_{i}$ and thus nonzero at the point (14). The non-singularity of the Jacobian ensures (15) via the implicit function theorem.

The main result can now be formulated.

Theorem 1 Let

$$
Z_{1, \nu_{1}}^{o}, \ldots, Z_{p, \nu_{p}}^{o}, V^{o}
$$

solve (13). Then, for any set of real analytic functions $u_{1}(t), . ., u_{p}(t)$ with

$$
U\left(t_{0}\right)=V^{o}
$$


there exists an $\epsilon>0$ such that, on the interval $\left(t_{0}-\epsilon, t_{0}+\epsilon\right)$, there are real solutions $z_{1}(t), . ., z_{n}(t)$ satisfying (10) with

$$
Z_{i}^{(\nu-1)}\left(t_{0}\right)=Z_{i, \nu-1}^{o}, \quad i=1, \ldots, n
$$

Proof. From Proposition 7 the equations of (10) are locally equivalent to

$$
\begin{aligned}
z_{1}^{\left(\nu_{1}\right)} & =\phi_{1}\left(Z_{1}^{\left(\nu_{1}-1\right)}, \ldots, Z_{n}^{\left(\nu_{n}-1\right)}, U\right) \\
\vdots & \\
z_{n}^{\left(\nu_{n}\right)} & =\phi_{n}\left(Z_{1}^{\left(\nu_{1}-1\right)}, \ldots, Z_{n}^{\left(\nu_{n}-1\right)}, U\right)
\end{aligned}
$$

with the initial conditions

$$
Z_{i}^{(\nu-1)}\left(t_{0}\right)=Z_{i, \nu-1}^{o}, \quad i=1, \ldots, n
$$

which can be converted to a state space description using the standard state assignment

$$
\begin{gathered}
x_{1}=z_{1}, x_{2}=\dot{z}_{1}, \ldots, x_{\nu_{1}}=z_{1}^{\left(\nu_{1}-1\right)} \\
x_{\nu_{1}+1}=z_{2}, x_{\nu_{1}+2}=\dot{z}_{2}, \ldots, x_{\nu_{1}+\nu_{2}}=z_{2}^{\left(\nu_{2}-1\right)}
\end{gathered}
$$

The existence of a local solution to (17) then follows from standard results on ordinary differential equations, see e. g. [14]. Since the inequations and inequalities are satisfied at $t_{0}$ they are satisfied on a small enough interval.

\section{A simple example}

Are there any real solutions to the following system?

$$
\begin{aligned}
\dot{y}_{1}^{2}+\dot{y}_{2}^{2}-1 & =0 \\
y_{1} y_{2}-1 & =0 \\
\ddot{y}_{1}+\ddot{y}_{2} & <0
\end{aligned}
$$

Running algorithm FG, for the ranking

$$
y_{1}<\dot{y}_{1}<\ddot{y}_{1}<\cdots<y_{2}<\dot{y}_{2}<\ddot{y}_{2}<\cdots
$$

together with reduction gives the following system

$$
\begin{aligned}
\left(y_{1}^{4}+1\right) \dot{y}_{1}^{2}-y_{1}^{4} & =0 \\
y_{1} y_{2}-1 & =0 \\
y_{1} & \neq 0 \\
y_{1}^{4}+1 & \neq 0 \\
\dot{y}_{1} & \neq 0 \\
y_{1}^{11}\left(y_{1}^{2}+1\right)\left(y_{1}^{4}+1\right)^{3} & <0
\end{aligned}
$$

The other systems created by splitting in algorithm FG trivially lack solutions. We see that any negative initial value of $y_{1}$ will lead to a solution satisfying the relations in (20). We thus have to solve the initial value problem

$$
\dot{y}_{1}= \pm \sqrt{y_{1}^{4} /\left(y_{1}^{4}+1\right)}, \quad y_{2}=1 / y_{1}
$$

with $y_{1}(0)<0$.

\section{Conclusions}

We have shown how one can decide algorithmically if a system (1) has a real solution. First algorithm FG is used to reduce the problem to the checking of a number of systems of the form (1) but where the equalities form an auto-reduced set whose separants and initials are among the $g_{i}$ and where the $g_{i}$ and $h_{i}$ are reduced. Since the equations are then equivalent to a set of explicit differential equations (17), it is enough to find an initial condition satisfying the equations, inequations and inequalities. This a problem of real algebra, which can be solved by known methods.

An natural generalization would be to allow also inequality constraints of the form $h_{i} \leq 0$. This would introduce new aspects into the problem, such as the possibility of choosing the $u$ to make the solution remain in the feasible region.

\section{References}

[1] D. S. Arnon, G. E. Collins, and S. McCallum. Cylindrical algebraic decomposition i: The basic algorithm. SIAM J. Comput., 13:865-889, 1984.

[2] D. S. Arnon, G. E. Collins, and S. McCallum. Cylindrical algebraic decomposition ii: An adjacency algorithm for the plane. SIAM J. Comput., 13:878-889, 1984.

[3] J. Bochnak, M. Coste, and M-F. Roy. Géometrie algébrique réelle. Springer-Verlag, 1987.

[4] S. Diop. A state elimination procedure for nonlinear systems. In J. Descusse, M. Fliess, A. Isidori, and D. Leborgne, editors, New trends in Nonlinear Control Theory, Lect. Notes Control Inform. Sci. 122, pages 190-198. Springer, 1989.

[5] S. Diop. Elimination in control theory. Math. Control Signals Systems, 4:17-32, 1991.

[6] M. Fliess and S. T. Glad. An algebraic approach to linear and nonlinear control. In H. L. Trentelman and J. C. Willems, editors, Essays on control: Perspectives in the theory and its applications., pages 223-267. Birkhäuser, 1993.

[7] M. Fliess and M. Hasler. Questioning the classical state space description via circuit examples. In M. A. Kaashoek, J. H. van Schuppen, and A. C. M. Ran, editors, Realization and Modelling in System Theory, pages 1-12. Birkhüser, 1990.

[8] S. T. Glad. Differential algebraic modelling of nonlinear systems. In M. A. Kaashoek, J. H. van Schuppen, and A. C. M. Ran, editors, Realization and Modelling in System Theory, pages 97-105. Birkhüser, 1990. 
[9] S. T. Glad. Implementing Ritt's algorithm of differential algebra. In IFAC Symposium on Control Systems Design, NOLCOS'92, pages 610614, Bordeaux, France, June 1992.

[10] Manfred Knebusch and Claus Scheiderer. Einführung in die reelle Algebra. Vieweg studium, 1989.

[11] E.R. Kolchin. Differential Algebra and Algebraic Groups. Academic Press, New York, 1973.

[12] J. F. Ritt. Differential Algebra. American Mathematical Society, Providence, R I, 1950.

[13] A. Seidenberg. An elimination theory for differential algebra. In F. Wolf, J. L. Hodges, and A. Seidenberg, editors, University of California Publications in Mathematics: New Series, pages 31-66. University of California Press, Berkeley and Los Angeles, California, 1956.

[14] F. Verhulst. Nonlinear differential equations and dynamical systems. Springer-Verlag, 1985. 